\title{
Original
}

\section{Simulation of Capillary Hemodynamics and Comparison with Experimental Results of Microphantom Perfusion Weighted Imaging}

\author{
Shurche S. ${ }^{\oplus}$, Riyahi Alam N.2,3,4*๑
}

\begin{abstract}
Background: Perfusion imaging, one of MRI's techniques, is widely used to test damaged tissues of the body. The parameters used in this technique include cerebral blood flow (CBF), cerebral blood volume (CBV), and mean transit time (MTT). The MRI scanner contains a device called a "phantom", which controls the accuracy of various imaging models.
\end{abstract}

Objective: Our goal is to design and produce a microphantom to control the perfusion-imaging model in MRI scanners.

Material and Methods: Firstly, in an analytical study type, we designed the phantom based on Murray's minimum work rule using AutoCAD software. Next, the phantom was fabricated using lithography and then imaged using a Siemens Magnetom 3T Prisma MRI scanner at the National Brain Laboratory. Finally, the velocity and pressure in the capillary network was simulated using COMSOL software.

Results: CBF, CBV, and MTT curves for the capillary network were obtained at different times. In addition, the simulations showed that the velocity and pressure in the capillary network were between 0.0001 and $0.0005 \mathrm{~m} / \mathrm{s}$ and between 5 and 25 $\mathrm{mm} / \mathrm{Hg}$, respectively.

Conclusion: The fabricated microphantom was used to simulate the movement of blood within tissues of the body. Different parameters of perfusion imaging were measured inside the phantom, and they in the phantom were similar to in the body.

Citation: Shurche S, Riyahi Alam N. Simulation of Capillary Hemodynamics and Comparison with Experimental Results of Microphantom Perfusion Weighted Imaging. J Biomed Phys Eng. 2020;10(3):291-298. doi: 10.31661/jbpe.v0i0.910.

\section{Keywords}

Perfusion Imaging; Phantoms, Imaging; Cerebral Blood Volume; Capillary Hemodynamics; Contrast Media

\section{Introduction}

hanges in cerebral perfusion are one symptom of chronic and acute illnesses in head diseases. Various theories propose that a capillary map can be used to diagnose the capillaries as a cause of illness and can contribute to the strategy for the treatment of the underlying disease [1]. Cerebral blood flow (CBF), cerebral blood volume $(\mathrm{CBV})$, and mean transit time (MTT) are used in the perfusion imaging and in examining damaged tissues of the body [2]. An MRI scanner and popular perfusion-imaging techniques such as dynamic susceptibility contrast (DSC) and arterial spin labeling (ASL) are used to mea-
${ }^{1} \mathrm{MSc}$ Student, Physics and Medical Engineering

Department, Medical

Faculty, Tehran Univer-

sity of Medical Sciences,

Tehran, Iran

${ }^{2} \mathrm{PhD}$, Department of

Medical Physics and

Biomedical Engineering,

Faculty of Medicine, Teh-

ran University of Medi-

cal Sciences (TUMS),

Tehran, Iran

${ }^{3}$ PhD, PERFORM Preven-

tive Medicine and Health

Care Center, Concordia

University, Montreal,

Quebec, Canada

${ }^{4} \mathrm{PhD}$, Medical Pharma-

ceutical Sciences Re-

search Center (MPRC),

The institute of Pharma-

ceutical Sciences, teh-

ran University of Medical

Sciences, Tehran, Iran

*Corresponding author: N. Riyahi Alam

Physics and Medical Engineering Department, Medical Faculty, Tehran University of Medical Sciences, P.O.Box: 1417613151, Tehran, Iran

E-mail: riahialam@gmail. com

Received: 6 February 2918 Accepted: 10 March 2019 
sure these parameters. However, both techniques, especially DSC, face many problems in computing various parameters of perfusion. Tremendous effort has been expended to find a mathematical model for the DSC method. Noise plays an important role in the instability of the parameters involved in the perfusion imaging and has a significant effect on thresholding. The signal-to-noise ratio (SNR) is a dependent parameter and bolus delay is a source of errors in the perfusion measurements. To measure these uncertainties and errors, perfusion models must be studied using phantoms with the same capillary network dimensions as in humans.

Various types of phantoms are used in the study of perfusion models [3], with the simplest providing detailed information while the most complicated may not be appropriate for testing perfusion models.

In this paper, we introduce a new phantom with a complex capillary network and with which perfusion parameters can be measured accurately. The capillary density of this phantom is similar to that of the human body and, therefore, can provide a CBV similar to that of the human body. The phantom was fabricated using lithographic techniques, the flexibility of which allows the simulation of capillary diseases such as stenosis. This phantom can be used to study perfusion model errors and enhance the perfusion-imaging technique.

\section{Material and Methods}

\section{Theory}

In this analytical study, most perfusion models consist of two basic models: the Kety Schmidt model [4] for penetrating contrast agents, and the Meier Zierler model for nonpenetrating contrast agents. These two models are the same in terms of computing, but they are essentially different from each other. In the following, we discuss the Meier Zierler model because we use a nonpenetrating contrast agent. First, the residue function is defined as
[5]:

$$
R(t)=1-\int_{0}^{t} h(t) d t
$$

Where $h$ is the vascular transport function. The density-time diagram of the tissue is related to the density-time diagram using the following convolution equation:

$C_{T}=F \int_{-\infty}^{t} C_{A}(t-\tau) R(\tau) d \tau=F\left(C_{A} \otimes R\right)$

Where $F$ is the flow rate, and is the same as $\mathrm{CBF}$, and $C_{\mathrm{A}}$ is the arterial input function. Equation (2) can be used to control perfusion measurements. To calculate the contrast agent the rate convolutions should be taken of the residue function and the arterial input function (AIF). CBV and MTT, two other parameters involved in perfusion are calculated as follows [6]:

$$
\begin{aligned}
& C B V=\frac{\int_{-\infty}^{\infty} C_{T} d t}{\int_{-\infty}^{\infty} C_{A} d t} \\
& M T T=\frac{C B V}{C B F}
\end{aligned}
$$

\section{Design of MicroPhantom}

In designing the phantom, we used the tree pattern used in quantitative studies on the cardiovascular network and Murray's minimum work rule [7]. To simulate the hemodynamics of the vessels (Figure 1). According to the minimum work rule, nature uses a minimum of four energies. Therefore, the relationship between the diameter and the bifurcation angle of the capillaries is [8]:

$$
\begin{aligned}
& \frac{r_{0}^{3}}{f_{0}}=\frac{r_{1}^{3}}{f_{1}}=\frac{r_{2}^{3}}{f_{2}}=\text { const } \\
& \cos \left(\theta_{1}\right)=\frac{r_{0}^{4}+r_{1}^{4}-r_{2}^{4}}{r^{4}{ }_{1} r_{2}}
\end{aligned}
$$




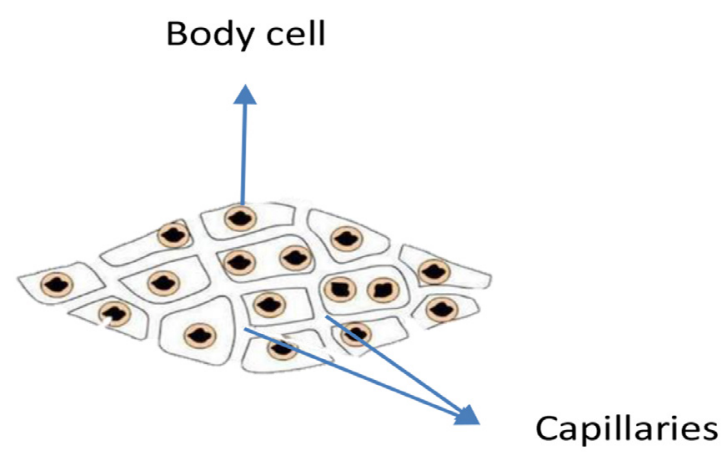

Figure 1: Shape of the capillary network inside the body.

$$
\cos \left(\theta_{2}\right)=\frac{r_{0}^{4}+r_{2}^{4}-r_{1}^{4}}{r^{4}{ }_{1} r^{4}{ }_{2}}
$$

Where $r_{0}, r_{1}$, and $r_{2}$ are the radii of the main and daughter channels, $\theta_{1}$ and $\theta_{2}$ are the bifurcation angles, and $f_{0}, f_{1}$, and $f_{2}$ are the flow in the main and daughter channels. Murray's rule is applicable for circular channel sections; however, owing to prospective difficulties in fabrication, we used rectangular sections [9]. Therefore, so as to be able to apply Murray's rule to rectangular sections, the following formula was used in our calculation and designs:

$\alpha_{n}\left(1+\alpha_{n}\right) P_{0}\left(\alpha_{n}^{*}\right)=(2 X)^{n} \alpha_{n}\left(1+\alpha_{0}\right) P_{0} \alpha_{0}^{*}$
Where $\alpha_{n}$ and $\mathrm{a}^{*}{ }_{n}$ are the ratio of the depth $d$ to the width $w$ of channel $n$ when the depth is smaller than the width.

The network has two major structural components: the supplying channels and the cells. The main channels are larger than the rest of the phantom sections that Murray's rule has been used in order to design them. Table 1 shows the width of main and daughter channels in Calculations and in the microphantom. The three main channels are outside the capillary network. However, four other sub channels are within it. Each block consists of eight cells that replace the capillary network in the body. The results of Pawlik et al. [10] and Cassot et al. [11] were used to calculate the size of each microchannel (see Figure 2).

Table 1: The width of main and daughter channels in Calculations and in the microphantom.

\begin{tabular}{ccc} 
Generation & Phantom $(\boldsymbol{\mu m})$ & Theory $^{*}(\boldsymbol{\mu m})$ \\
\hline 1 & 5038.345 & 1085 \\
\hline 2 & 2887.98 & 597 \\
\hline 3 & 1850.08 & 353 \\
\hline 4 & 298.011 & 230 \\
\hline 5 & 71 & 25
\end{tabular}

${ }^{*}$ Calculated using Eq. (8)

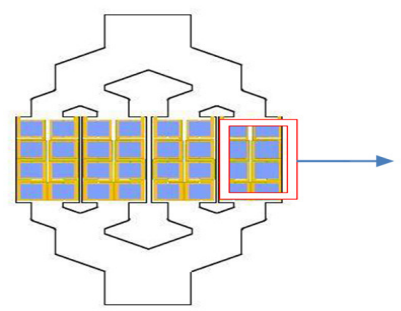

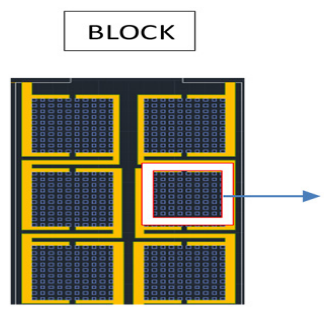

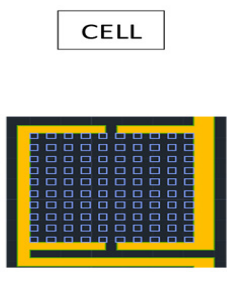

Figure 2: (a) Fabricated microphantom design and enlargement of the layout of the "blocks" and "cells". The phantom is $15.506 \mathrm{~mm}$ wide. The microphantom consists of four blocks, each block also contains eight cells, and each cell has 100 features separated by channels, which are only $71 \mu \mathrm{m}$ wide. Contrast agent passes through these channels. 
According to the theories, different calculations of the capillary network were performed. Then, using the parameters obtained from the calculations, the capillary network was designed using AutoCAD software (M.49.0.0 AutoCAD 2016, Autodesk, Inc., San Rafael, CA, USA) (see Figure 2).

\section{Microfabrication}

We used a soft lithography technique to make the microphantom. This technique is called the soft lithography due to the use of a polymeric mold that gets soft and melting soon. To reproduce with soft lithography, you must first create a mold or seal. The most common way for it is to lithograph on a silicon surface $(101.6 \mathrm{~mm})$. This process creates an early pattern with prominent light-emitting designs that protrude from the silicon surface. Then, the polymeric fluid (SU-8, 2100 (MicroChem Corp., Boston, MA)) is poured into this highlight and turns into a soft, solid rubber. As a result, a polymeric manicure is made that has a perfect paradigm. The shape on the stamp differs by a few $\mathrm{nm}$ in the original pattern. Although the creation of an early pattern with outstanding elegant designs is costly, replicating the pattern on polymer seals is cheap and easy. Polymer seal (polydimethyl-siloxane (PDMS)) can be used to make nanostructures.

One of the soft lithographic methods is the printing method by the micrometric contact. Polymer sealing is impregnated with an effective solution, including organic molecules called thiol (in the role of ink). Then the stamp is strung across a thin film of gold (in the role of paper) that is placed on the glass, silicon or polymeric plates. The thiol reacts with the gold surface and creates a perfectly neat film that has the seamless design. Due to the diffusion of the ink after contacting with the surface, the precision of the single layer cannot be as large as the polymer seals. When used directly, a small $50 \mathrm{~nm}$ shape can be created (Figure 3).

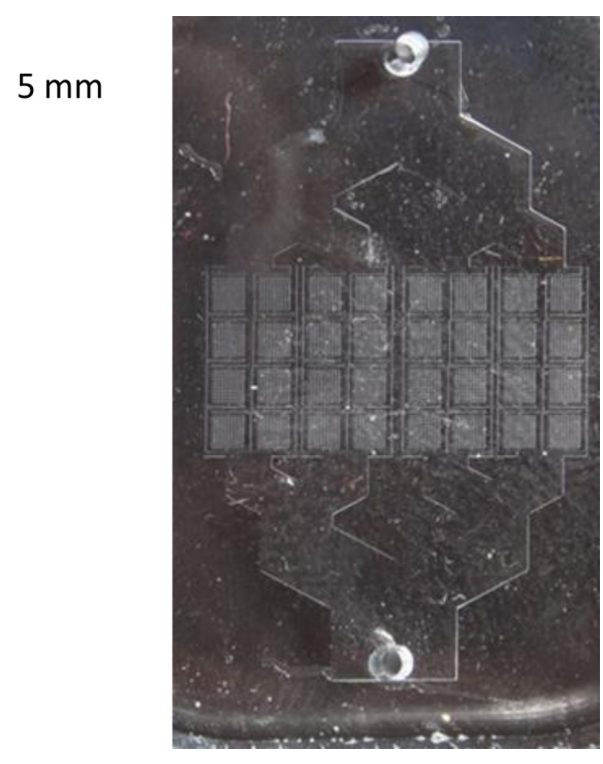

Figure 3: Microphantom fabricated using lithographic techniques. The two holes on the top and bottom of the phantom are for the injection (entrance) and exit of water or contrast agent.

\section{Experimental setup}

Distilled water was injected into the capillary network using a pump connected to the microphantom cavity via a connector. Subsequently, the microphantom was placed in the middle of the rat coil and $300 \mu 1$ of distilled water was injected each time. The measurement methods used has been described here. A gradient spin-echo pulse sequence with the following parameters was used for the perfusion measurements in $3 \mathrm{~T}$ :

The echo time $(\mathrm{TE})=5 \mathrm{~ms}$, the repetition time $(\mathrm{TR})=860 \mathrm{~ms}(1650 \mathrm{~ms}$ after two times averaging), field of view $=6 \mathrm{~cm} \times 6 \mathrm{~cm}$ and the resolution was $32 \times 32$ pixels.

The amount of $\mathrm{CBV}$ within the phantom has a different definition, which is equal to the division of the desired microchannel volume into the total voxel volume in that area.

Contrast agent, injection rate was considered in injector $0.00216-0.023 \mathrm{~mL} / \mathrm{s}$. By calculating the volume of the capillary network and 
taking into account the density of its material, this injection rate of the contrast material causes the rate of movement of the contrast media within the tissue to be equal to $0.2-1.75$ $\mathrm{mL} / 100 \mathrm{gr} / \mathrm{s}$.

\section{Simulation}

The Navier Stokes equation was used to calculate the flow for the entire capillary network. The boundary conditions at the walls were considered zero in the software used to solve the equation. The capillary network designed using AutoCAD software was transferred to the COMSOL Multiphysics ${ }^{\circledR}$ software version 5.3 (COMSOL, Inc., Burlington, MA, USA) and simulated. From the simulation of the map, we obtained the capillary velocity map and used it to calculate the flow of the stream.

\section{Results}

\section{Flow measurement}

The MRI image of the phantom is shown in Figure 4.

The CBV was calculated using Eq. (3) and is shown in Figure 5. With the value of CBV and knowing the value of MTT, we can obtain the value of CBF from Eq. (4).

\section{Flow Simulation}

The velocity map of the capillary network, obtained using the COMSOL simulation software and presented in Figure 6, shows that the velocity in the capillary network was between 0.0001 and $0.0005 \mathrm{~m} / \mathrm{s}$.

The pressure map of the capillary network, also obtained using the COMSOL simulation software, is shown in Figure 7. The highest pressure (red) was on the interstitial side of the capillary network and decreased as the capillary mesh output was approached (blue). The pressure in the capillary network was between 5 and $25 \mathrm{~mm} / \mathrm{Hg}$.

\section{Discussion}

The simulated velocity in the capillary network was between 0.0001 and $0.0005 \mathrm{~m} / \mathrm{s}$ (Figure 6), which agreed with the results of Ivanov et al. [12].

The simulated pressure in the capillary network was between 5 and $25 \mathrm{~mm} / \mathrm{Hg}$ (Figure 7 ), which was consistent with the results of Shore et al [13].

These results reveal that our phantom can be used to create conditions similar to the blood

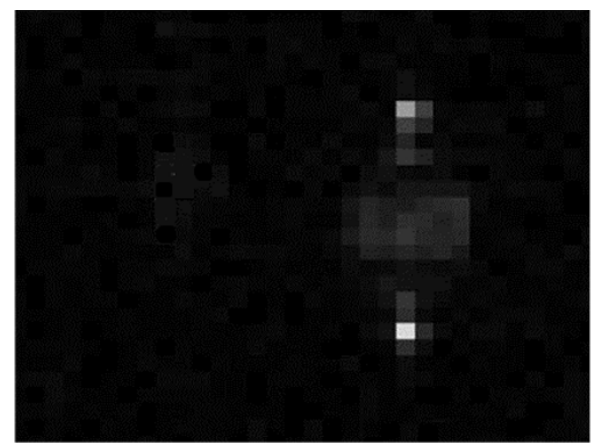

a

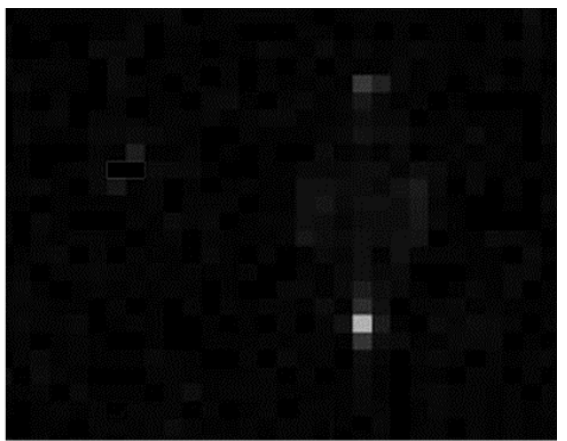

b

Figure 4: (a) Fabricated microphantom design and enlargement of the layout of the "blocks" and "cells". The phantom is $15.506 \mathrm{~mm}$ wide. The microphantom consists of four blocks, each block also contains eight cells, and each cell has 100 features separated by channels, which are only $71 \mu \mathrm{m}$ wide. Contrast agent passes through these channels. 

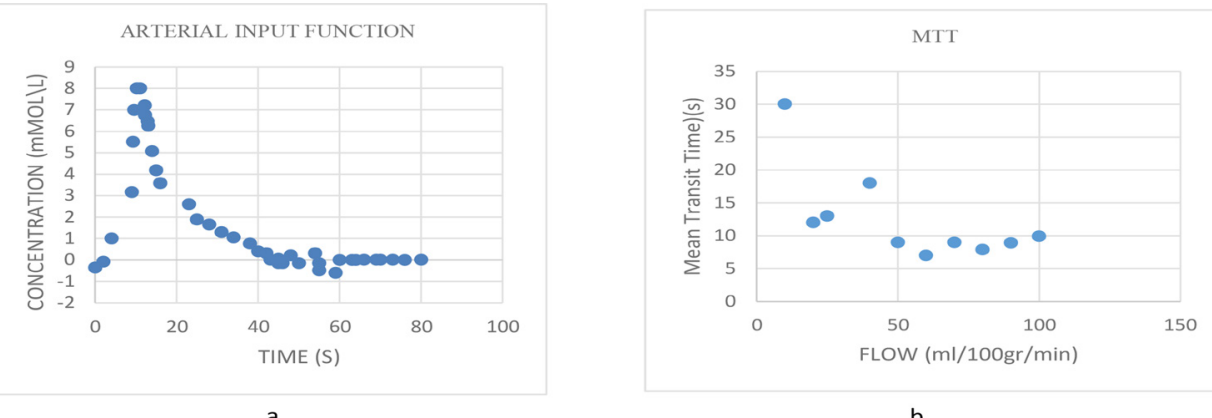
b

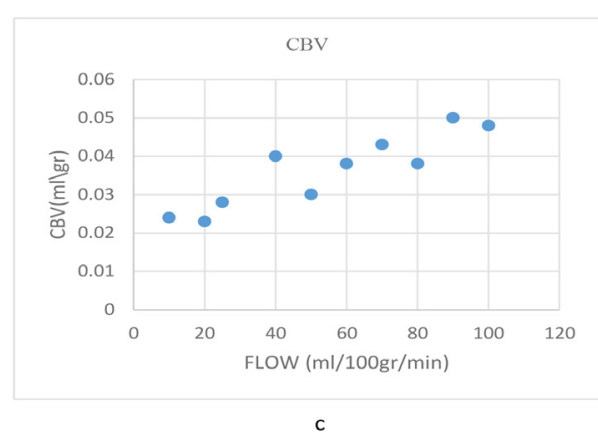

Figure 5: Values of AIF (a), CBV(b) and MTT(c) measured in the microphantom.
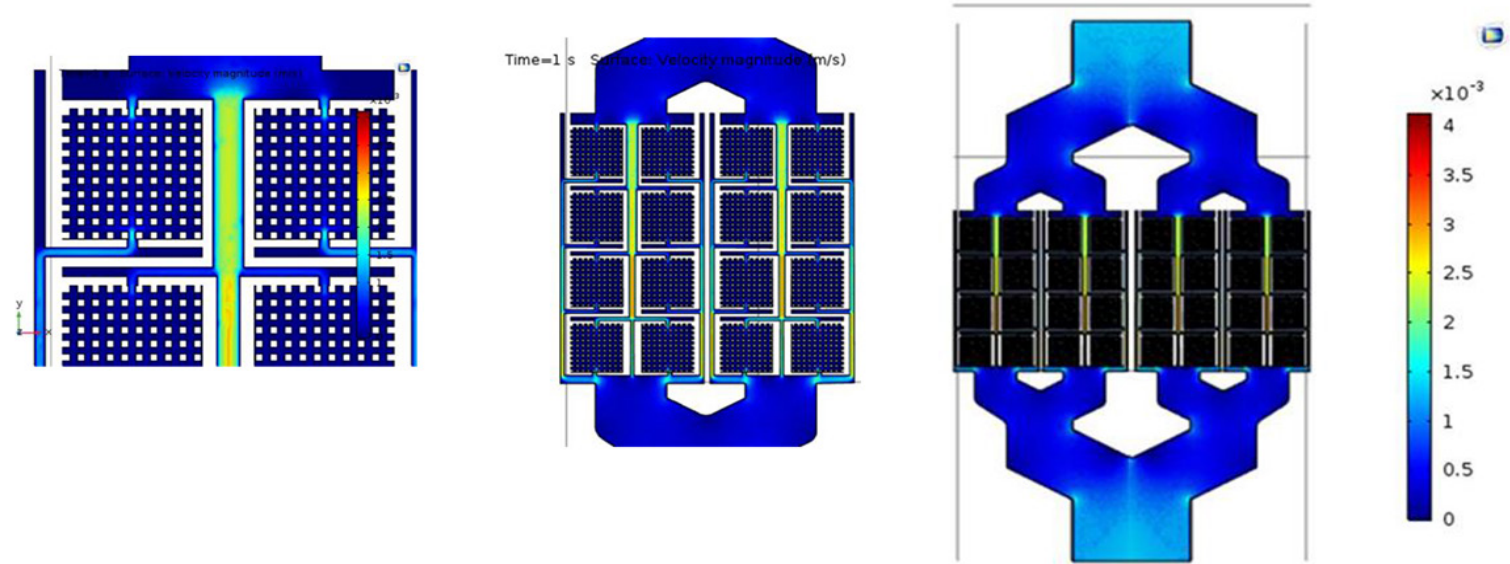

C

b

a

Figure 6: Velocity map for the capillary network obtained using COMSOL simulation software (version 5.3). The highest velocity was found in the capillary network.

flow in the body and to examine various models of perfusion imaging with an MRI scanner. Different parameters of perfusion imaging had the similar value to the inside of the body [14].

\section{Conclusion}

A phantom for perfusion measurement using MRI is presented. With this approach, any network geometry can be designed. The 


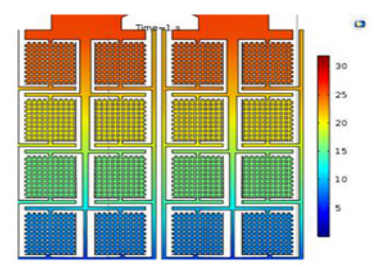

C
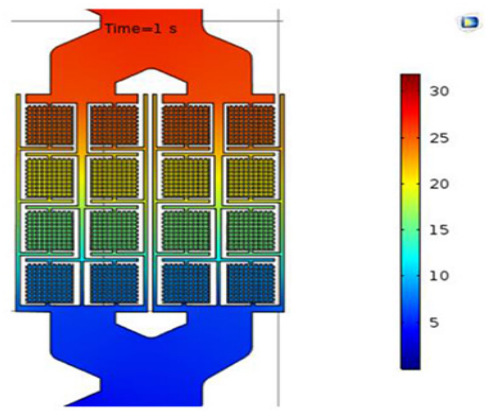

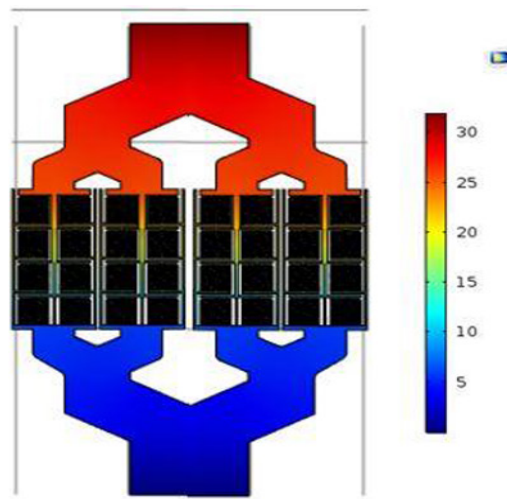

a

Figure 7: Pressure map of the capillary network obtained using COMSOL simulation software (version 5.3). The highest pressure (red) was on the interstitial side of the capillary network and decreased as the capillary mesh output was approached (blue).

channels are in the range of actual capillaries. The phantom is MR compatible and flow rates, everywhere in the sample can be calculated precisely to be compared with estimated flow rates to investigate the accuracy of models. Purely PDMS phantoms are used for nondiffusible and diffusible tracers, respectively. According to the results, this phantom can be used to validate various methods in perfusion imaging such as singular value decomposition deconvolution.

\section{Acknowledgment}

The authors thank Tehran University of Medical Sciences (TUMS) for funding this work. The results of this study were extracted from a thesis funded by TUMS (grant No. 9413338003). The authors also thank the staff of the University of Sharif Microelectronics Laboratory, and the National Brain Laboratory (NBML) for collaborate on making and microphantom imaging, and access to the Amir Kabir Cloud Computing Portal (HP$\mathrm{CRC}$ ) for simulation. The simulation was performed under Contract No. ISI-DCE-DODCloud-700101-1990 at Amir Kabir University.

\section{Conflict of Interest}

$$
\text { None }
$$

\section{References}

1. Ostergaard L, Aamand R, Gutierrez-Jimenez E, Ho YC, Blicher JU, Madsen SM, et al. The capillary dysfunction hypothesis of Alzheimer's disease. Neurobiol Aging. 2013;34:1018-31. doi: $\quad$ 10.1016/j.neurobiolaging.2012.09.011. PubMed PMID: 23084084.

2. Wintermark M, Sesay M, Barbier E, Borbely K, Dillon WP, Eastwood JD, et al. Comparative overview of brain perfusion imaging techniques. Stroke. 2005;36:e83-99. doi: 10.1161/01.STR.0000177884.72657.8b. PubMed PMID: 16100027.

3. Kato H, Kuroda M, Yoshimura K, Yoshida A, Hanamoto K, Kawasaki S, et al. Composition of MRI phantom equivalent to human tissues. Med Phys. 2005;32:3199-208. doi: 10.1118/1.2047807. PubMed PMID: 16279073.

4. Kety SS, Schmidt CF. The determination of cerebral blood flow in man by the use of nitrous oxide in low concentrations. American Journal of Physiology-Legacy Content. 1945;143:5366.

5. Meier P, Zierler KL. On the theory of the indicator-dilution method for measurement of blood flow and volume. J Appl Physiol. 1954;6:73144. doi: 10.1152/jappl.1954.6.12.731. PubMed PMID: 13174454.

6. Copen WA, Schaefer PW, Wu O. MR perfusion imaging in acute ischemic stroke. Neuroimag- 
Shurche S and Riahi Alam N.

ing Clin N Am. 2011;21:259-x. doi: 10.1016/j. nic.2011.02.007. PubMed PMID: 21640299. PubMed PMCID: PMC3135980.

7. Murray CD. The Physiological Principle of Minimum Work: I. The Vascular System and the Cost of Blood Volume. Proc Natl Acad Sci U S A. 1926;12:207-14. PubMed PMID: 16576980. PubMed PMCID: PMC1084489.

8. Rossitti S, Lofgren J. Vascular dimensions of the cerebral arteries follow the principle of minimum work. Stroke. 1993;24:371-7. PubMed PMID: 8446972.

9. Emerson DR, Cieslicki K, Gu X, Barber RW. Biomimetic design of microfluidic manifolds based on a generalised Murray's law. Lab Chip. 2006;6:447-54. doi: 10.1039/b516975e. PubMed PMID: 16511629.

10.Pawlik G, Rackl A, Bing RJ. Quantitative capillary topography and blood flow in the cerebral cortex of cats: an in vivo microscopic study. Brain Res. 1981;208:35-58.
11.Cassot F, Lauwers F, Fouard C, Prohaska S, Lauwers-Cances $V$. A novel three-dimensional computer-assisted method for a quantitative study of microvascular networks of the human cerebral cortex. Microcirculation. 2006;13:118. doi: 10.1080/10739680500383407. PubMed PMID: 16393942.

12.Ivanov KP, Kalinina MK, Levkovich Yu I. Blood flow velocity in capillaries of brain and muscles and its physiological significance. Microvasc Res. 1981;22:143-55. PubMed PMID: 7321902.

13.Shore AC. Capillaroscopy and the measurement of capillary pressure. $\mathrm{Br} J$ Clin Pharmacol. 2000;50:501-13. PubMed PMID: 11136289. PubMed PMCID: PMC2015012.

14.Calamante F, Morup M, Hansen LK. Defining a local arterial input function for perfusion MRI using independent component analysis. Magn Reson Med. 2004;52:789-97. doi: 10.1002/ mrm.20227. PubMed PMID: 15389944. 